\title{
Eptifibatide induced acute profound thrombocytopenia and compartment syndrome during the treatment course of ST-segment elevation myocardial infarction
}

\author{
Meng Kuang Lee ${ }^{1}$, Wen-Hsien Lee ${ }^{1}$, Chen-An Chiu ${ }^{1}$, Ho-Ming Su ${ }^{1,2}$, Tsung-Hsien Lin ${ }^{1,}{ }^{2}$, \\ Wen-Chol Voon ${ }^{1,2}$, Wen-Ter Lai ${ }^{1,2}$, Sheng-Hsiung Sheu ${ }^{1,2}$, Po-Chao Hsu ${ }^{1,2,3}$ \\ 1. Division of Cardiology, Department of Internal Medicine, Kaohsiung Medical University Hospital, Kaohsiung Medical \\ University, Kaohsiung, Taiwan. 2. Department of Internal Medicine, School of Medicine, Kaohsiung Medical University, \\ Kaohsiung, Taiwan. 3. Graduate Institute of Medicine, School of Medicine, Kaohsiung Medical University, Kaohsiung, \\ Taiwan.
}

Correspondence: Po-Chao Hsu. Address: Division of Cardiology, Department of Internal Medicine; Kaohsiung Medical University Hospital, 100 Tzyou 1st Road, Kaohsiung. 80708, Taiwan. Email: pochao.hsu@gmail.com

Received: April 3, 2014

DOI : $10.5430 /$ crim.v1n2p111

Online Published: May 9, 2014

Accepted: April 29, 2014

im.v1n2p111

\begin{abstract}
Eptifibatide is an anti-platelet drug which belongs to a kind of glycoprotein IIb/IIIa receptor inhibitors and it is used to decrease ischemic events while treating acute coronary syndromes. However, eptifibatide-related acute profound thrombocytopenia (APT) was rarely reported and could lead to increased mortality. We present a case experiencing APT after eptifibatide infusion which resulted in compartment syndrome of the left hand. To our knowledge, this patient was the first case of eptifibatide-related thrombocytopenia complicated with compartment syndrome of the forearm. This rare case reminds physicians that compartment syndrome is an extremely rare but still possible complication after GPIIb/IIIa inhibitors use. In these patients, we should discontinue GPIIb/IIIa inhibitor infusion and correct thrombocytopenia with platelet transfusion as soon as possible. Surgical intervention may not be the only choice if the compartment syndrome is gradually improved under intensive medical care.
\end{abstract}

\section{Keywords}

Compartment syndrome, Eptifibatide, ST elevation myocardial infarction, Thrombocytopenia

\section{Introduction}

Eptifibatide is an anti-platelet drug which belongs to a kind of glycoprotein (GP) IIb/IIIa receptor inhibitors. It inhibits the binding of fibrinogen to the platelet fibrinogen receptor, and therefore inhibits platelet aggregation and thrombus formation. It is used to decrease ischemic events while treating acute coronary syndromes ${ }^{[1]}$. Because of its antiplatelet effect, acute thrombocytopenia (platelet counts $<100000 / \mu \mathrm{L}$ ) is a well-known complication. However, acute profound thrombocytopenia (APT) with platelet count $<20000 / \mu \mathrm{L}$ is a rare but potentially lethal complication which may became a challenge to physician ${ }^{[2]}$. Herein we present a case experiencing acute profound thrombocytopenia after eptifibatide infusion which resulted in compartment syndrome of the left hand. To our knowledge, this patient was the first case of eptifibatide-related thrombocytopenia complicated with compartment syndrome of the forearm, and we report the management and clinical course of this rare adverse reaction. 


\section{Case report}

A 77-year-old male presented with acute chest pain, cold sweating and dyspnea for 5 hours and then visited our emergency department (ED). Tracing back his history, he had the underlying disease of hypertension with regular medication control. On arrival at our ED, initial blood pressure was $121 / 77 \mathrm{mmHg}$, and heart rate was 102 beats per minute. The electrocardiography (ECG) revealed ST segment elevation in II, III and aVF leads with reciprocal ST segment depression in I, aVL, and V2 to V4 leads (see Figure 1). In addition, there was no ST segment elevation over V3R and V4R in right side ECG. Complete blood cell (CBC) counts showed white blood cell count at 10800/uL, hemoglobin level at $14.2 \mathrm{~g} / \mathrm{dL}$ and the platelet count at 191000/uL. The cardiac enzymes showed creatinine kinase (CK) at $298 \mathrm{IU} / \mathrm{L}, \mathrm{CK}-\mathrm{MB}$ at 25.2 $\mathrm{ng} / \mathrm{mL}$, and Troponin-I at $1.41 \mathrm{ng} / \mathrm{mL}$. The prothrombin time (PT) and activated partial thromboplastin time (aPTT) level were all within normal limits. The ST-segment elevation myocardial infarction was recognized in the patient. After administrating $300 \mathrm{mg}$ of clopidogrel, 300mg of aspirin, bolus infusion of $4000 \mathrm{U}$ heparin and further continuous infusion of heparin pump (12U/kg/hour), coronary angiography was immediately arranged. The procedure was performed via left transradial approach. Right coronary angiogram revealed $99 \%$ stenosis with large amount thrombus formation over right coronary artery (RCA) (see Figure 2A). Left coronary angiogram revealed $80 \%$ stenosis over middle left anterior descending artery and total occlusion over proximal left circumflex artery (see Figure 2B). As RCA was the most favored culprit vessel according to ECG and angiographic findings, we decided to perform primary percutaneous coronary intervention (pPCI) of RCA. Intravenous bolus infusion of eptifibatide was given and thrombus aspiration was performed several times by using aspiration catheter. However, there was still large burden of thrombus noted after thrombus aspiration and further balloon angioplasty. We finally discontinued PCI and kept continuous infusion of eptifibatide with 2 $\mathrm{ug} / \mathrm{kg} / \mathrm{min}$. The final result of right coronary angiography showed only TIMI 1 flow to distal RCA with residual large thrombus burden. Radial sheath was removed without complications and then the patient was transferred to our intensive care unit (ICU).

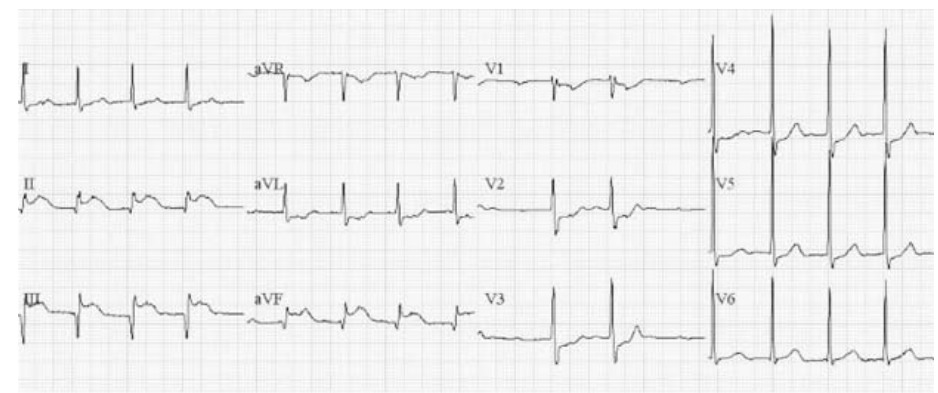

Figure 1. Electrocardiogram showed ST segment elevation in leads II,III and aVF.
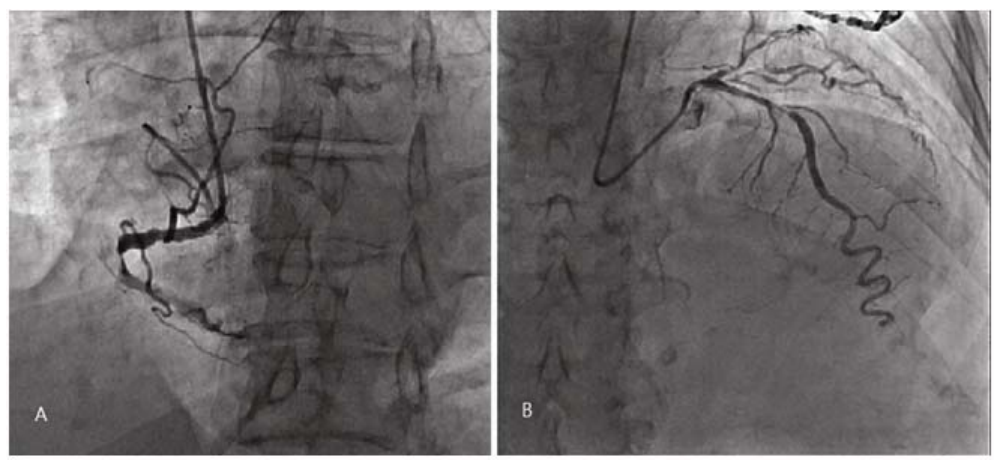

Figure 2A. Right coronary angiogram revealed a $99 \%$ stenosis at proximal right coronary artery with large thrombus burden. Figure 2B. Left coronary angiogram revealed $80 \%$ stenosis at middle left descending artery and total occlusion at proximal left circumflex artery 
During ICU stay, initially no obvious hematoma in puncture site was noted after removing radial sheath, but left arm swelling with bullae formation was gradually noted 6 hours after admission to ICU (see Figure 3). Follow-up of laboratory data revealed severe thrombocytopenia $(6000 / \mu \mathrm{L})$ with normal disseminated intravascular coagulation (DIC) profile. We immediately stopped eptifibatide infusion and transfused the patient with 6 units of platelet concentrate. The patient's left hand was becoming more and more cyanotic and distal pulse on radial and ulnar artery gradually could not be palpated, so we arranged emergent computed tomography (CT) to evaluate the cause and range of critical limb ischemia. The result of $\mathrm{CT}$ revealed the suspicion of distal segment of brachial artery occlusion with poor collateralization and some hematoma formation in forearm. We also consulted with cardiovascular surgeons to evaluate the situation but they did not favor surgical intervention because of no more progression of compartment syndrome and limited neural deficits of the left hand. Therefore, we kept intensive care and observation for the patient. After several days of follow-up, there was no further deterioration of the left hand ischemia and compartment syndrome also gradually improved after platelet transfusion and intensive medical care. Platelet counts were completely normalized after one week (see Figure 4). Due to severe coronary artery disease (three-vessel disease) the patient underwent coronary artery bypass graft surgery which was performed 20 days after admission. The patient was discharged at the 41 th day of hospitalization.

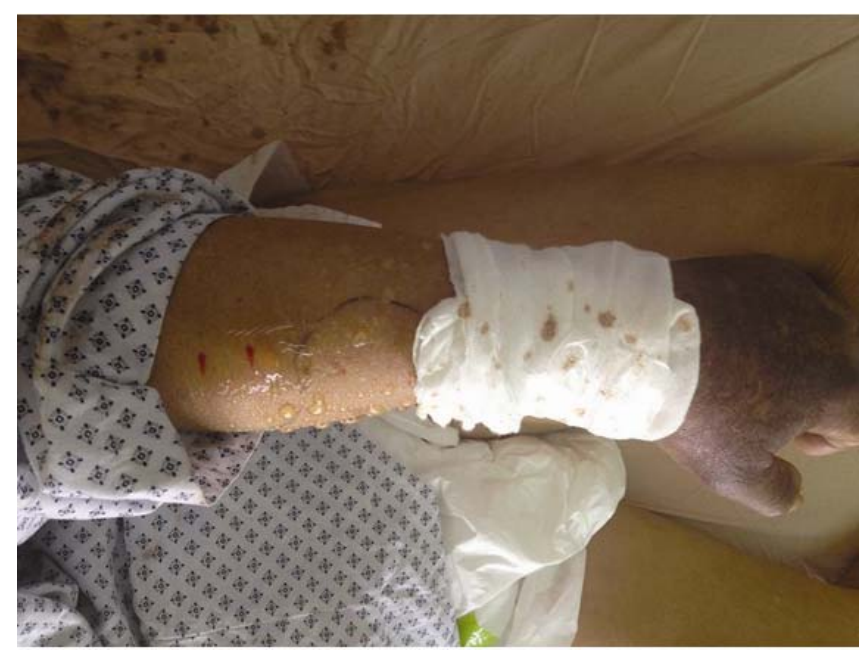

Figure 3. Left arm swelling with multiple bullae formation was gradually noted after admission to intensive care unit.

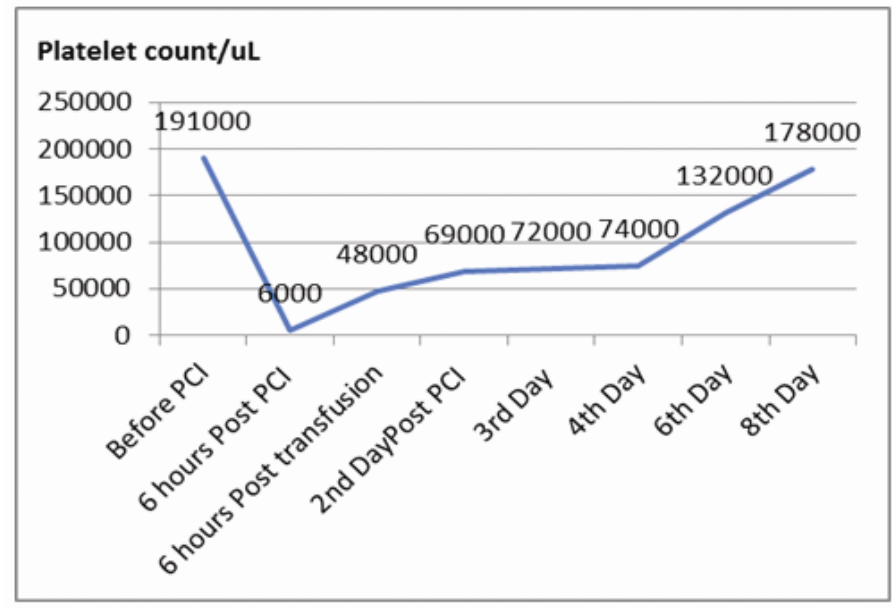

Figure 4. The amount of platelet counts during hospitalization. (PCI: percutaneous coronary intervention) 


\section{Discussion}

The GPIIb/IIIa inhibitors act by blocking the binding of fibrinogen to activated GPIIb/IIIa, and therefore inhibiting the platelet-platelet interaction for thrombus formation. Nowadays GPIIb/IIIa inhibitors are used widely in the treatment of acute coronary syndromes ${ }^{[3,4]}$.Three kinds of GPIIb/IIIa inhibitor agents: abciximab, tirofiban and eptifibatide are applied in everyday practice. Thrombocytopenia and bleeding risk are most common complications after these agents' use. Eptifibatide, a second class of GPIIb/IIIa of ligand-mimetic agents, was found to have less thrombocytopenia event in compare to abciximab. Thrombocytopenia events associated with eptifibatide occurred in $6.8 \%$ of the patients, while APT was reported to occur in only $0.2 \%$ which can cause severe hemorrhagic event ${ }^{[5,6]}$. The mechanism of thrombocytopenia in eptifibatide use can be caused by immune or nonimmune factors ${ }^{[6]}$.

Because we treated the patient with heparin and eptifibatide simultaneously, heparin-induced thrombocytopenia (HIT) had to be excluded. HIT typically occurs during 5 to 14 days after initiation of heparin administration. It rarely happened after 2 weeks. Sometimes, early onset of HIT (median time of platelet decreased after heparin injection) was noted in patients experienced heparin administration in the previous one to three months. In addition, HIT behaves severe rarely. Platelet counts are usually 50000 to $70000 / \mu \mathrm{L}$. Platelet counts $<20000 / \mu \mathrm{L}$ is infrequently and often accompanied with DIC ${ }^{[7]}$. In our case, the patient has never experienced heparin administration before this hospitalization and the platelet count decreased immediately within only 6 hours. DIC profile was also negative. Therefore, HIT was not favored in our patient.

The management of GPIIb/IIIa inhibitors induced APT is remained unclearly defined. Immediately discontinue of GPIIb/IIIa inhibitors was necessary. Platelet transfusion to patients whose platelet counts $<20000 / \mu \mathrm{L}$ is needed because of the potential risk of active bleeding. However; platelet transfusion until platelet counts $<10000 / \mu \mathrm{L}$ had also been proved safe for patients without active bleeding ${ }^{[8,9]}$. Corticosteroids and intravenous immunoglobulin (IVIG) was used in idiopathic thrombocytopenic purpura but very limited data was noted to treat GPIIb/IIIa induced thrombocytopenia ${ }^{[9]}$. Therefore, we only discontinued eptifibatide infusion and gave platelet transfusion to correct APT in our patient.

GPIIb/IIIa inhibitors induced APT resulting in compartment syndrome of the forearm was rarely reported and we only found one case reported in the literature ${ }^{[10]}$. Oh YJ et al. ever reported a case of abciximab induced APT which resulted in compartment syndrome of the forearm from massive bleeding at the brachial artery access site ${ }^{[10]}$. However, our case was different from their case in several points. First, GPIIb/IIIa inhibitor which we used was eptifibatide but not abciximab. Second, the cause of compartment syndrome was also different between the two cases. In their patient, puncture site was over brachial artery which caused further massive bleeding due to APT. However, puncture site in our case was in radial artery and we did not find massive bleeding from the CT examination despite somewhat hematoma in the left forearm. The most possible mechanism of compartment syndrome in our case might be associated with pPCI performed over left hand which caused vessel injury and further eptifibatide-induced APT which caused blood extravasation. According to the literature, the interruption of the normal interaction between endothelial cells and platelets is clinically visible in patients with severe thrombocytopenia, in whom platelet-membrane exposure and release of endothelial-cell trophogens is reduced to the point at which the multimolecular vascular-endothelium cadherin complex disassembles, resulting in the loss of the intercellular barrier and red blood cells extravasation into the tissues ${ }^{[11]}$. To our knowledge, our patient is the first described case of eptifibatide-induced APT complicated with compartment syndrome of the forearm.

\section{Conclusion}

We reported a case of the patient experiencing compartment syndrome - the APT complication after administration of epifibitate. This rare case reminds physicians that compartment syndrome is an extremely rare but still possible complication after GPIIb/IIIa inhibitors use. In these rare patients we should discontinue GPIIb/IIIa inhibitor and correct thrombocytopenia via blood transfusion as soon as possible. Surgical intervention may not be the only choice if the compartment syndrome is gradually improved under intensive medical supervision. 


\section{References}

[1] Karvouni E, Katritsis DG, Ioannidis J. Intravenous glycoprotein IIb/IIIa receptor antagonists reduce mortality after percutaneous coronary interventions. J Am Coll Cardiol. 2003; 41: 26-32. http://dx.doi.org/10.1016/S0735-1097(02)02666-9

[2] Hongo RH, Brent BN. Association of eptifibatide and acute profound thrombocytopenia. Am J Cardiol. 2001; 88: $428-31$. http://dx.doi.org/10.1016/S0002-9149(01)01695-2

[3] O'Gara PT, Kushner FG, Ascheim DD, Casey DE Jr, Chung MK, de Lemos JA, et al. American College of Cardiology Foundation/American Heart Association Task Force on Practice Guidelines. 2013 ACCF/AHA Guideline for the Management of ST-Elevation Myocardial Infarction: A Report of the American College of Cardiology Foundation/American Heart Association Task Force on Practice Guidelines. Circulation. 2013; 127: e362-e425. http://dx.doi.org/10.1161/CIR.0b013e3182742c84

[4] Wright RS, Anderson JL, Adams CD, Bridges CR, Casey DE Jr, Ettinger SM, et al. American College of Cardiology Foundation/American Heart Association Task Force on Practice Guidelines. 2011 ACCF/AHA Focused Update Incorporated Into the ACC/AHA 2007 Guidelines for the Management of Patients With Unstable Angina/Non-ST-Elevation Myocardial Infarction. J Am Coll Cardiol. 2011; 57: e215-367. http://dx.doi.org/10.1016/j.jacc.2011.02.011

[5] THE PURSUIT TRIAL INVESTIGATORS. Inhibition of platelet glycoprotein IIb/IIIa with eptifibatide in patients with acute coronary syndromes N Engl J Med. 1998; 339: 436-443. http://dx.doi.org/10.1056/NEJM199808133390704

[6] Richard H. Aster, MD. Immune Thrombocytopenia Caused by Glycoprotein IIb/IIIa Inhibitors. Chest. 2005; 127(suppl 2): 53S-59S.

[7] Lee GM, Arepally GM. Heparin-induced thrombocytopenia Hematology Am Soc Hematol Educ Program. 2013;2013:668-74.

[8] Wandt H, Frank M, Ehninger G, Schneider C, Brack N, Daoud A, Fackler-Schwalbe I, et al. Safety and cost effectiveness of a $10 \times$ $109 / \mathrm{L}$ trigger for prophylactic transfusions compared with the traditional $20 \times 109 / \mathrm{L}$ triggera prospective comparative trial in 105 patients with acute myeloid leukemia ,Blood. 1998; 91: 3601-3606.

[9] Huxtable LM, Tafreshi MJ, Rakkar AN. Frequency and Management of Thrombocytopenia With the Glycoprotein IIb/IIIa Receptor Antagonists. Am J Cardiol. 2006 Feb 1; 97(3): 426-9. Review. http://dx.doi.org/10.1016/j.amjcard.2005.08.066

[10] Oh YJ, Park TH, Choi YJ, Cho SH, Choi JH, Lee DH, et al. Abciximab (ReoPro)-Induced Thrombocytopenia Diagnosed Through Measurement of Heparin-Dependent Antibody. Korean Circ J. 2009 Feb; 39(2): 75-78. http://dx.doi.org/10.4070/kcj.2009.39.2.75

[11] Nachman RL, Rafii S. Platelets, petechiae, and preservation of the vascular wall. N Engl J Med. 2008 Sep 18; 359 (12): 1261-70. http://dx.doi.org/10.1056/NEJMra0800887 